N. Toda

Nagoya Math. J.

Vol. 34 (1969), 1-23

\title{
SUR LES DIREGTIONS DE JULIA ET DE BOREL DES FONCTIONS ALGEBROIDES
}

\author{
NOBUSHIGE TODA
}

\section{Introduction.}

L'extension aux fonctions algébroïdes du théorème de Picard est faite par Rémoundos [7] et après quelques années, celle de la théorie de Nevanlinna aux fonctions algébroïdes dans $|z|<+\infty$ est donnée par Selberg [9], Ullrich [14] et Valiron [16]. Dans ce mémoire, on considère l'allure des fonctions algébroïdes d'après la méthode dernière.

Soient $f(z)$ une fonction algébroïde dans $|z|<+\infty$ définie par

$$
f^{n}+a_{1}(z) f^{n-1}+\cdots+a_{n}(z)=0
$$

où $a_{1}(z), \cdots, a_{n}(z)$ sont méromorphes dans $|z|<+\infty$ et au moins une desquels n'est pas rationnelle, $R_{f}$ la surface de Riemann définie par $f(z)$ comme surface de recouvrement du plan fini, $R_{f}(r)$ la partie de $R_{f}$ sur $|z|<r$, $n(r, w)$ le nombre des pôles de $f(z)(w=\infty)$ ou des racines de l'équation $f(z)-w=0(w \neq \infty)$ sur $R_{f}(r)$ en consideration de son ordre de multiplicité, (et ainsi de suite),

$$
\begin{aligned}
& N(r, w)=\frac{1}{n} \int_{0}^{r} \frac{n(t, w)-n(0, w)}{t} d t+\frac{n(0, w)}{n} \log r \\
& m(r, w)= \begin{cases}\frac{1}{2 n \pi} \int_{\Gamma_{r}} \log ^{+}\left|\frac{1}{f\left(r e^{i \theta}\right)-w}\right| d \theta, & w \neq \infty, \\
\frac{1}{2 n \pi} \int_{\Gamma_{r}} \log ^{+}\left|f\left(r e^{i \theta}\right)\right| d \theta, & w=\infty,\end{cases}
\end{aligned}
$$

où $\Gamma(r)$ la frontière de $R_{f}(r), T(r, f)=N(r, \infty)+m(r, \infty), T(r, w)=N(r, w)+$ $m(r, w)(w \neq \infty), n\left(r, R_{f}\right)$ le nombre des points ramifiés sur $R_{f}(r)$ en considerant l'ordre du branche (et ainsi de suite) et

$$
N\left(r, R_{f}\right)=\frac{1}{n} \int_{0}^{r} \frac{n\left(t, R_{f}\right)-n\left(0, R_{f}\right)}{t} d t+\frac{n\left(0, R_{f}\right)}{n} \log r .
$$

Received March 28, 1968 
Alors, comme théorèmes fondamentals, on sait:

[I] Pour tout $w, T(r, w)=T(r, f)+O(1)$.

(Selberg [9]).

[II] Soient $a_{1}, a_{2}, \cdots, a_{q} q(\geqq 3)$ points sur la sphère de Riemann, alors sauf un ensemble de mesure linéaire finie de $r$,

$$
(q-2) T(r, f) \leqq \sum_{i=1}^{q} N\left(r, a_{i}\right)+N\left(r, R_{f}\right)+O(\log r T(r, f))
$$

(Selberg [9]).

$$
\left.N\left(r, R_{f}\right) \leqq(2 n-2) T(r, f)+O(1) \quad \text { (Ullich }[14]\right) .
$$

On dit qu'une valeur $a$ telle que l'ordre de $N(r, a)$ est moins de celui de $f(z)$ est valeur exceptionnelle au sens de Borel, où l'ordre de $f(z)$ est $\lim _{r \rightarrow \infty} \sup \log T(r, f) / \log r$.

[IV] Si $f(z)$ est d'ordre positif, $f(z)$ admet au plus $2 n$ valeurs exceptionnelles au sens de Borel (Rémoundos [7]).

On definie les directions de Julia et de Borel pour $f(z)$ comme suivant (Voir [18]):

1) On dit que $J: \arg z=\theta$ est une direction de Julia pour $f(z)$ si $f(z)$ admet au plus $2 n$ valeurs exceptionnelles au sens de Picard dans $\Delta_{\varepsilon}(\theta)=\{z ;|\arg z-\theta|<\varepsilon\}$ où $\varepsilon$ est un nombre positif quelconque.

2) On dit que $B: \arg z=\alpha$ est une direction de Borel pour $f(z)$ si $f(z)$ admet au plus $2 n$ valeurs exceptionnelles au sens de Borel dans $\Delta_{\varepsilon}(\alpha)=\{z$; $|\arg z-\alpha|<\varepsilon\}$ où $\varepsilon$ est un nombre positif quelconque, quand l'ordre de $f(z)$ est positif. Une valeur $a$ telle que l'ordre de $N\left(r, a ; \Delta_{\varepsilon}\right)$ est inférieur à celui de $f(z)$ est dite valeur exceptionnelle au sens de Borel dans $\Delta_{\varepsilon}\left(=\Delta_{\varepsilon}(\alpha)\right)$.

$$
N\left(r, a ; \Delta_{\varepsilon}\right)=\frac{1}{n} \int_{0}^{r} \frac{n\left(t, a ; \Delta_{\varepsilon}\right)-n\left(0, a ; \Delta_{\varepsilon}\right)}{t} d t+\frac{n\left(0, a ; \Delta_{\varepsilon}\right)}{n} \log r
$$

où $n\left(r, a ; \Delta_{\varepsilon}\right)$ est le nombre des pôles de $f(z)$ dans $\Delta_{\varepsilon}(a=\infty)$ ou des zéros de $f(z)-a=0$ dans $\Delta_{\varepsilon}(a \neq \infty)$.

Pour les fonctions méromorphes dans $|z|<+\infty$, la discussion concernant les directions de Julia et de Borel est faite presque complètement par Julia [3], Ostrowski [4], Valiron [15], Biernacki [1], Rauch [5] et etc. . Mais pour les fonctions algébroïdes, il n'y a pas de résultats importants sauf deux résultats suivants: 
THÉORÈmE DE RAUCH ([6]). Soit $f(z)$ une fonction algébroìde entière d'ordre fini $\rho>0$ définie par l'équations (1). Alors, il existe au moins une direction d'ordre $\rho$ pour les racines de $f(z)-w=0$ sauf peut-être pour un ensemble de mesure linéaire nulle de valeur $w$. ("Entière" veut dire que $a_{1}, \cdots, a_{n}$ sont tous fonctions entières.)

ThÉORÈme DE VALIRon ([18]). Si $f(z)$ définie par (1) est d'ordre infini, alors il existe une direction de Borel pour $f(z)$.

Dans ce mémoire, on considère le suivant: Dans le paragraphe 2, on donne un exemple d'une fonction algébroïde qui n'a pas de direction de Julia. Le paragraphe 3 donne quelques conditions suffisantes pour que $f(z)$ ait une direction de Borel. Dans le paragraphe 4, on donne une amélioration du Théorème de Rauch. Dans le paragraphe 5 , on donne un théorème pour une fonction algébroïde qui admet l'ensemble d'adhérence fine d'éléments d'un nombre fini et dans le paragraphe 6 une condition suffisante pour qu'une fonction algébroïde ait la propriété de Wiman. Dans le paragraphe 7 , on donne des résultats analogues à ceux dans les paragraphes 3 et 4 pour les algébroïdes dans $|z|<1$.

Qu'il me soit permis pour terminer cette introduction d'exprimer ici ma reconnaissance à $M$. le Professeur Noshiro qui m'encourageait et à M. le Professeur Matsumoto auprès de qui $\mathrm{j}$ 'ai trouvé une aide constante pendant la préparation de ce travail.

\section{Un exemple d'une fonction algébroïde qui n'a pas de direc-} tion de Julia.

On donne ici un exemple d'une fonction algébroïde qui n'a pas de direction de Julia.

Soit

$$
a(z)=\frac{\prod_{n=0}^{\infty}\left(1-z / q^{n}\right)}{\prod_{n=0}^{\infty}\left(1+z / q^{n}\right)} \quad \text { où } \quad q>1 \text {, }
$$

alors, $a(z)$ est une fonction méromorphe dans $|z|<+\infty$ et exceptionnelle de Julia ([4]). Cette fonction admet les propriétés suivantes:

1) $|a(z)|=1$ sur l'axe imaginaire,

2) $|a(z)|<1$ pour $\operatorname{Re} z>0$ et $|a(z)|>1$ pour $\operatorname{Re} z<0$, et 
3) il existe deux nombres positifs $m$ et $M$ tels que

$$
0<m \leqq|a(z)| \leqq M<+\infty
$$

dans $\Delta=\left\{z ;\left|\arg z-\frac{\pi}{2}\right|<\theta\right\}$ où $0<\cos \theta<1 / 3$.

Soit $f(z)=\sqrt[n]{a(z)}$. Alors, par les propriétés 1), 2) et 3), il est facile à voir que $f(z)$ n'a pas de direction de Julia. Il faut démontrer 1), 2) et 3). 1) et 2) sont facile à voir. On démontre ici la propriété 3 ).

On sait l'inégalité

$$
|1-u| \geqq e^{-2 u} \quad \text { si } \quad|u|<1 / 2 .
$$

Deux produits $\prod_{n=0}^{\infty}\left(1-z / q^{n}\right)$ et $\prod_{n=0}^{\infty}\left(1+z / q^{n}\right)$ convergent absolument et uniformément au sens large dans $|z|<+\infty$, par conséquent on peut changer l'ordre de produit dans $|z| \leqq r<+\infty$ pour $r$ positif quelconque. Pour la brièveté, on évalue $|a(z)|^{2}$ dans $\Delta$. Soit $z=r(\cos \theta+i \sin \theta), 0<\theta<\frac{\pi}{2}$, alors

$$
\left|\frac{1-z / q^{n}}{1+z / q^{n}}\right|^{2}=\frac{r^{2}-2 r q^{n} \cos \theta+q^{2 n}}{r^{2}+2 r q^{n} \cos \theta+q^{2 n}}=1-\frac{4 r q^{n} \cos \theta}{r^{2}+2 r q^{n} \cos \theta+q^{2 n}},
$$

par conséquent,

$$
|a(z)|^{2}=\prod_{n=0}^{\infty}\left(1-\frac{4 r q^{n} \cos \theta}{r^{2}+2 r q^{n} \cos \theta+q^{2 n}}\right) .
$$

Pour $\theta$ tel que $\cos \theta<1 / 3$, on a

$$
\frac{4 r q^{n} \cos \theta}{r^{2}+2 r q^{n} \cos \theta+q^{2 n}}<1 / 2
$$

de sorte que, d'après l'inégalité (2), il est suffisant pour démontrer la propriété 3) de voir que la série

$$
s=\sum_{n=0}^{\infty} \frac{4 r q^{n} \cos \theta}{r^{2}+2 r q^{n} \cos \theta+q^{2 n}}
$$

est convergente.

Soit $n_{0}>1$ tel que $q^{n_{0}-1} \leqq r \leqq q^{n_{0}} \quad\left(\right.$ si $\left.r \leqq 1, n_{0}=1\right)$. On divise la série comme suivant:

$$
\begin{aligned}
s=\sum_{n=0}^{\infty} \frac{4 r q^{n} \cos \theta}{r^{2}+2 r q^{n} \cos \theta+q^{2 n}} & =\sum_{n=0}^{n_{0}-1} \frac{4 r q^{n} \cos \theta}{r^{2}+2 r q^{n} \cos \theta+q^{2 n}} \\
& +\sum_{n=n_{0}}^{\infty} \frac{4 r q^{n} \cos \theta}{r^{2}+2 r q^{n} \cos \theta+q^{2 n}} .
\end{aligned}
$$


En estimant les deux termes, on a

le premier terme $\leqq \sum_{n=0}^{n_{0}-1} \frac{4 r q^{n} \cos \theta}{r^{2}}=\frac{4 \cos \theta}{r} \cdot \sum_{n=0}^{n_{0}-1} q^{n}=\frac{4 \cos \theta}{r} \cdot \frac{1-q^{n_{0}}}{1-q}$

$$
=\frac{4 \cos \theta}{q-1}\left(\frac{q^{n_{\theta}}}{r}-\frac{1}{r}\right) \leqq \frac{4 q \cos \theta}{q-1}
$$

et

le second terme $\leqq \sum_{n=n_{0}}^{\infty} \frac{4 r q^{n} \cos \theta}{q^{2 n}}=4 r \cos \theta \cdot \sum_{n=n_{0}}^{\infty} \frac{1}{q^{n}}=4 r \cos \theta \cdot \frac{1}{q^{n_{\theta}}} \cdot \frac{1}{1-\frac{1}{q}}$

$$
=\frac{4 r \cos \theta}{q^{n_{0}}} \cdot \frac{q}{q-1} \leqq \frac{4 q \cos \theta}{q-1}
$$

c'est-à-dire

$$
s \leqq \frac{8 q \cos \theta}{q-1},
$$

en conséquence,

$$
|a(z)|^{2} \geqq \exp \left(-\frac{16 q \cos \theta}{q-1}\right) \geqq \exp \left(-\frac{16 q}{3(q-1)}\right),
$$

de cela, on a l'inégalité

$$
\exp \left(-\frac{8 q}{3(q-1)}\right) \leqq|a(z)| \leqq \exp \left(\frac{8 q}{3(q-1)}\right)
$$

dans $\Delta$ facilement.

3. Conditions suffisantes pour que des algébroïdes aient des directions de Borel.

On ne sait pas à mon regret s'il y a des directions de Borel pour les algébroïdes $f(z)$ d'ordre positif ou non en général. Dans ce paragraphe, on donne quelques conditions suffisantes pour qu'une fonction algébroïde dans $|z|<+\infty$ d'ordre $\rho>0$ admette au moins une direction de Borel.

Lemme 1. Soit $g(z)$ une fonction méromorphe dans $|z|<+\infty$ d'ordre fini $\rho>0$. Alors, il existe une direction $J: \arg z=\theta_{0}$ telle que pour $\delta>0$ quelque soit petit, dans $\Delta_{\delta}\left(\theta_{0}\right)=\left\{z ;\left|\arg z-\theta_{0}\right|<\delta\right\}$ le fait suivant est réalisé: Si $h(z)$ est une fonction méromorphe dans $|z|<+\infty$ d'ordre inférieur à $\rho$, alors

$$
\sum_{\nu=1}^{\infty} \frac{1}{\left|z_{\nu}\left(g=h: \Delta_{\delta}\right)\right|^{\rho-\varepsilon}}=+\infty
$$

pour tout $\varepsilon$ positif plus petit que $\rho$ avec deux exceptions possibles pour $h(z)$ (Biernacki [1]) et si $g(z)$ est de type divergent et $\int^{\infty} \frac{T(r, h)}{r^{\rho+1}} d r<+\infty$, alors, 


$$
\sum_{\nu=1}^{\infty} \frac{1}{\left|z_{\nu}\left(g=h ; \Delta_{\delta}\right)\right|^{\rho}}=+\infty
$$

avec deux exceptions possibles pour $h(z)$ (Rauch [5]); où $z_{\nu}\left(g=h ; \Delta_{\delta}\right)(\nu=1,2$, $3, \cdots)$ sont les zéros de $g(z)-h(z)$ dans $\Delta_{\delta}\left(\theta_{0}\right)$ sans considération de multiplicité. (Voir [12].)

THÉORÈme 1. Soit $f(z)$ une fonction algébroïde d'ordre fini $\rho>0$ définie par (1). Si un seul des $a_{1}(z), a_{2}(z), \cdots, a_{n}(z)$ est d'ordre $\rho$ et d'autres sont d'ordre inférieur à $\rho$, il existe une direction $J: \arg z=\theta_{0}$ telle que $f(z)$ satisfait à la relation

$$
\sum_{\nu=1}^{\infty} \frac{1}{|z(a)|^{\rho-\varepsilon}}=+\infty
$$

pour tout $\varepsilon$ positif plus petit que $\rho$ sauf au plus $2 n$ valeurs de a; de plus quand $f(z)$ est de type divergent, si un seul des coefficients est de type divergent d'ordre $\rho$ et d'autres sont de type convergent d'ordre $\rho$ au plus, on peut changer (5) comme

$$
\sum_{\nu=1}^{\infty} \frac{1}{|z(a)|^{\rho}}=+\infty
$$

où $\left\{z_{\nu}(a)\right\}_{\nu=1}^{\infty}$ sont les zéros de $f(z)-a$ ou les pôles de $f(z)$ si $a=\infty$ dans $\Delta_{\delta}\left(\theta_{0}\right)=\left\{z ;\left|\arg z-\theta_{0}\right|<\delta\right\}$ où $\delta>0$ quelconque.

Démonstration. a) Le cas où au moins deux coefficients ne sont pas constants.

Soit $a_{k}(z)$ le coefficient de (1) d'ordre $\rho$. D'après le lemme 1 , les zéros de $a_{k}(z)-h(z)$ satisfont à (4) sauf au plus deux $h(z)$ d'ordre inférieur à $\rho$. Soit

$$
h_{\alpha}(z)=-\frac{\alpha^{n}+a_{1}(z) \alpha^{n-1}+\cdots+a_{k-1}(z) \alpha^{n-k+1}+a_{k+1}(z) \alpha^{n-k-1}+\cdots+a_{n}(z)}{\alpha^{n-k}},
$$

$\alpha \neq 0$, alors $h_{\alpha}(z)$ est d'ordre inférieur à $\rho$ pour $\alpha \neq 0$ et

$$
\left\{z ; a_{k}(z)-h_{\alpha}(z)=0\right\}=\left\{z ; \alpha^{n}+a_{1}(z) \alpha^{n-1}+\cdots+a_{n}(z)=0\right\},
$$

c'est-à-dire, les zéros de $f(z)-\alpha$ coïncident avec les zéros de $a_{k}(z)-h_{\alpha}(z)$. Par conséquent, en appliquant le lemme 1, sauf deux $h_{\alpha_{1}}$ et $h_{\alpha_{2}}$ au plus, les zéros de $a_{k}(z)-h_{\alpha}(z)$ satisfont à (4). Or, le nombre de $\beta(\neq \alpha)$ tel que $h_{\alpha}(z)=h_{\beta}(z)$ est au plus $n-2$. En effet, supposons qu'il existe $n-1$ valeurs $\beta_{1}, \beta_{2}, \cdots, \beta_{n-1}$. Soit 


$$
\begin{aligned}
& \frac{\alpha^{n}+a_{1}(z) \alpha^{n-1}+\cdots+a_{k-1}(z) \alpha^{n-k+1}+a_{k+1}(z) \alpha^{n-k-1}+\cdots+a_{n}(z)}{\alpha^{n-k}} \\
= & \frac{\beta_{i}^{n}+a_{1}(z) \beta_{i}^{n-1}+\cdots+a_{k-1}(z) \beta_{i}^{n-k+1}+a_{h+1}(z) \beta_{i}^{n-k-1}+\cdots+a_{n}(z)}{\beta_{i}^{n-k}} \\
= & -h(z) \quad(i=1,2,3, \cdots, n-1),
\end{aligned}
$$

alors,

$$
\begin{array}{r}
\alpha^{n}+a_{1}(z) \alpha^{n-1}+\cdots+h(z) \alpha^{n-k}+\cdots+a_{n}(z)=0, \\
\beta_{i}^{n}+a_{1}(z) \beta_{i}^{n-1}+\cdots+h(z) \beta_{i}^{n-k}+\cdots+a_{n}(z)=0 \\
(i=1,2,3, \cdots, n-1) .
\end{array}
$$

De ces $n$ équations, $a_{1}(z), \cdots, a_{k-1}(z), a_{k+1}(z), \cdots, a_{n}(z)$ et $h(z)$ reduisent aux constants. C'est une contradiction à l'hypothèse. Par conséquent, il existe au plus $2(n-1)+2=2 n$ (le " 2 " est pour 0 et $\infty$ ) valeurs qui ne satisfont pas à (5). On peut démontrer le reste de la même façon en appliquant la dernière partie du lemme 1.

b) Le cas où un seul coefficient n'est pas constant.

Soit $a_{k}(z)$ le coefficient d'ordre $\rho$. Les zéros de $a_{k}(z)-\alpha$ satisfont à (4) sauf deux $\alpha$ au plus. Soient $\alpha_{1}$ et $\alpha_{2}$ deux valeurs exceptionnelles possibles. Les racines des équations

$$
\begin{aligned}
& w^{n}+a_{1}(z) w^{n-1}+\cdots+\alpha_{1} w^{n-k}+\cdots+a_{n}(z)=0, \\
& w^{n}+a_{1}(z) w^{n-1}+\cdots+\alpha_{2} w^{n-k}+\cdots+a_{n}(z)=0
\end{aligned}
$$

sont au plus $2 n$. Par conséquent, sauf ces $2 n$ valeurs au plus, toutes les autres satisfont à (5). Le reste de ce cas est aussi démontré de la même manière en appliquant la dernière partie du lemme 1.

N.B. La direction $J$ de ce théorème est une direction de Borel pour $f(z)$.

ThÉORÈme 2. Soit $f(z)$ une fonction algébroïde d'ordre $\rho>0$. S'il existe $n-1$ valeurs exceptionnelles formelles finies, alors il $y$ a une direction de Borel pour $f(z)$. Ici, une valeur u exceptionnelle formelle finie veut dire que l'ordre de

$$
F(u, z)=u^{n}+a_{1}(z) u^{n-1}+\cdots+a_{n}(z)
$$

est inférieur à $\rho$. 
Démonstration. Soient $u_{1}, u_{2}, u_{3}, \cdots, u_{n-1}(n-1)$ valeurs exceptionnelles formelles finies. Alors,

$$
F\left(u_{i}, z\right)=u_{i}^{n}+a_{1}(z) u_{i}^{n-1}+\cdots+a_{n}(z)=g_{i}(z) \quad(i=1,2, \cdots, n-1)
$$

sont d'ordre inférieur à $\rho$.

Soit $u$ une valeur finie qui n'est pas égale aux $u_{1}, u_{2}, \cdots, u_{n-1}$, alors

$$
F(u, z)=u^{n}+a_{1}(z) u^{n-1}+\cdots+a_{n}(z)=g(z)
$$

est d'ordre $\rho$. Résolvons ces équations par rapport aux $a_{1}(z), a_{2}(z), \cdots$, $a_{n-1}(z)$ et $a_{n}(z)$, alors, on a

$$
a_{j}(z)=\alpha_{j} g(z)+h_{j}(z), \quad(j=1,2,3, \cdots, n),
$$

où $\alpha_{j}$ est un constant et $h_{j}(z)$ est une fonction méromorphe d'ordre inférieur à $\rho(j=1,2,3, \cdots, n)$. L'équation (1) reduit à

$$
w^{n}+h_{1}(z) w^{n-1}+\cdots+h_{n}(z)+\left(\alpha_{1} w^{n-1}+\cdots+\alpha_{n}\right) g(z)=0 .
$$

Or, il existe au plus $n$ valeurs $w$ telles que, pour une fonction $B(z)$ méromorphe dans $|z|<+\infty$,

$$
\frac{w^{n}+h_{1}(z) w^{n-1}+\cdots+h_{n}(z)}{\alpha_{1} w^{n-1}+\cdots+\alpha_{n}} \equiv B(z) .
$$

En effet, soient $v_{1}, v_{2}, \cdots, v_{n}, v_{n+1}$ telles valeurs différentes. Alors,

$$
\begin{aligned}
h_{1}(z) v_{j}^{n-1}+\cdots+h_{n}(z)=\left(\alpha_{1} v_{j}^{n-1}+\cdots+\alpha_{n}\right) B(z)-v_{j}^{n}, \\
\\
(j=1,2, \cdots, n+1) .
\end{aligned}
$$

En résolvant les premières $n$ équations par rapport aux $h_{1}(z), h_{2}(z), \cdots$, $h_{n}(z)$, on a

$$
h_{j}(z)=\alpha_{j} B(z)+\beta_{j} \quad(j=1,2, \cdots, n)
$$

où $\beta_{j}$ est un constant. Par conséquent

$$
\frac{w^{n}+h_{1}(z) w^{n-1}+\cdots+h_{n}(z)}{\alpha_{1} w^{n-1}+\cdots+\alpha_{n}}=\frac{w^{n}+\beta_{1} w^{n-1}+\cdots+\beta_{n}}{\alpha_{1} w^{n-1}+\cdots+\alpha_{n}}+B(z) .
$$

C'est-à-dire que $v_{1}, v_{2}, \cdots, v_{n}, v_{n+1}$ sont les racines de l'équation

$$
w^{n}+\beta_{1} w^{n-1}+\cdots+\beta_{n}=0 \text {. }
$$

C'est une contradiction, parce que l'équation algébrique de degré $n$ admet au plus $n$ racines distinctes. 
En utilisant ce fait, grâce au lemme 1 , on a ce théorème en considérant que

$$
\{z ; f(z)-a=0\}=\left\{z ; g(z)-\frac{a^{n}+h_{1}(z) a^{n-1}+\cdots+h_{n}(z)}{\alpha_{1} a^{n-1}+\cdots+\alpha_{n}}=0\right\}
$$

immédiatement.

Corollaire 1. Il existe une direction de Borel pour les fonctions algébroïdes d'ordre $\rho>0$ qui admet $2 n$ valeurs exceptionnelles au sens de Borel.

Le point ramifié n'a pas d'influence dans les discussions précédentes. Ici, on considère le cas où il y influe.

Lemme 2. Soient $f(z)$ une fonction algébroïde dans $|z|<1$ à k branches, $R_{f}$ la surface de recouvrement sur $|z|<1$ définie par $f(z)$ et $a_{1}, a_{2}, \cdots, a_{q} q(\geqq 3)$ points de w-sphère. Alors, si le nombre d'éléments de $\left\{p \in R_{f} ; f(p)=a_{i}, i=1\right.$ ou 2 ou $\cdots$ ou q\} est $n_{1}$ et le nombre des points ramifiés sur $R_{f}$ est $n_{2}$, on a

$$
(q-2) S(r) \leqq n_{1}+n_{2}+\frac{A}{1-r}
$$

ou

$$
S(r)=\frac{1}{\pi} \iint_{x(r)}\left(\frac{\left|f^{\prime}\left(r e^{i \varphi}\right)\right|}{1+\left|f\left(r e^{i \varphi}\right)\right|^{2}}\right)^{2} r d r d \varphi,
$$

$\chi(r)$ la partie de $R_{f}$ sur $|z|<r$ et $A$ est un constant dépendant de $a_{1}, a_{2}, \cdots, a_{q}$ seulement.

Démonstration. D'après une extension du théorème fondamental d'Ahlfors concernant la surface de recouvrement par Dufresnoy [2], Tumura [13] et Sario-Noshiro [8], on a

$$
(q-2) S(r) \leqq n_{1}+n_{2}+h L(r)
$$

où

$$
L(r)=r \int_{\Gamma(r)} \frac{\left|f^{\prime}\left(r e^{i \varphi}\right)\right|}{1+\left|f\left(r e^{i \varphi}\right)\right|^{2}} d \varphi,
$$

$\Gamma(r)$ la frontière de $\chi(r)$ et $h$ est un constant ne dépendant que de $w$-sphère moins $\left\{a_{1}, a_{2}, \cdots, a_{q}\right\}$.

1) Le cas où $(q-2) S\left(r^{\prime}\right)-n_{1}-n_{2}>0$ pour tout $r^{\prime}$ plus grand que $r$. En utilisant l'inégalité 


$$
L(r)^{2} \leqq \pi r \frac{d S(r)}{d r}
$$

on a

$$
\left\{(q-2) S\left(r^{\prime}\right)-n_{1}-n_{2}\right\}^{2} \leqq h^{2} L\left(r^{\prime}\right)^{2} \leqq h^{2} \pi r^{\prime} \frac{d S\left(r^{\prime}\right)}{d r^{\prime}}
$$

par conséquent

$$
\begin{aligned}
1-r<\int_{r}^{1} \frac{d r^{\prime}}{r^{\prime}} & \leqq \frac{h^{2} \pi}{(q-2)^{2}} \cdot \int_{r}^{1} \frac{d S\left(r^{\prime}\right)}{\left\{S\left(r^{\prime}\right)-\frac{n_{1}+n_{2}}{q-2}\right\}^{2}} \\
& \leqq \frac{h^{2} \pi}{(q-2)^{2}} \cdot \frac{1}{S(r)-\frac{n_{1}+n_{2}}{q-2}},
\end{aligned}
$$

c'est-à-dire

$$
(q-2) S(r)<n_{1}+n_{2}+\frac{A}{1-r}
$$

où $A=h^{2} \pi /(q-2)$.

2) Le cas où il existe un $r^{\prime}$ plus grand que $r$ tel que $(q-2) S\left(r^{\prime}\right)-n_{1}$ $-n_{2}<0$.

On a immédiatement l'inégalité

$$
(q-2) S(r) \leqq(q-2) S\left(r^{\prime}\right)<n_{1}+n_{2}<n_{1}+n_{2}+\frac{A}{1-r} .
$$

Lemme 3. Soient $f(z)$ une fonction algébroide dans $\Delta_{0}=\left\{z ;|\arg z| \leqq \alpha_{0}\right\}$ à $k$ branches, $R_{f}$ la surface de recouvrement sur $\Delta_{0}$ définie par $f(z), \Delta=\{z ;|\arg z| \leqq \alpha\}$ où $0<\alpha<\alpha_{0}, \quad \Delta_{0}(r)=\Delta_{0} \cap(|z|<r), \quad \Delta(r)=\Delta \cap(|z|<r), \quad R_{f}^{0}(r)$ ou $R_{f}(r)$ la partie de $R_{f}$ sur $\Delta_{0}(r)$ ou $\Delta(r)$ respectivement,

$$
\begin{gathered}
S(r, f ; \Delta)=S(r, \Delta)=\frac{1}{\pi} \iint_{R_{f}(r)}\left(\frac{\left|f^{\prime}\left(r e^{i \varphi}\right)\right|}{1+\left|f\left(r e^{i \varphi}\right)\right|^{2}}\right)^{2} r d r d \varphi, \\
T(r, f ; \Delta)=T(r, \Delta)=\frac{1}{k} \int_{0}^{r} \frac{S(r, \Delta)}{r} d r,
\end{gathered}
$$

$n\left(r, a ; \Delta_{0}\right)$ le nombre des zéros de $f(z)-a$ ou des pôles de $f(z)$ si $a=\infty$ sur $R_{f}^{0}(r)$,

$$
N\left(r, a ; \Delta_{0}\right)=\frac{1}{k} \int_{0}^{r} \frac{n\left(r, a ; \Delta_{0}\right)-n\left(0, a ; \Delta_{0}\right)}{r} d r+\frac{n\left(0, a ; \Delta_{0}\right)}{k} \log r,
$$


$n\left(r, R_{f} ; \Delta_{0}\right)$ le nombre des points ramifiés de $R_{f}(r)$ et

$$
N\left(r, R_{f} ; \Delta_{0}\right)=\frac{1}{k} \int_{0}^{r} \frac{n\left(r, R_{f} ; \Delta_{0}\right)-n\left(0, R_{f} ; \Delta_{0}\right)}{r} d r+\frac{n\left(0, R_{f} ; \Delta_{0}\right)}{k} \log r,
$$

alors on a

$$
(q-2) S(r, \Delta) \leqq 3 \sum_{i=1}^{q} n\left(2 r, a_{i} ; \Delta_{0}\right)+3 n\left(2 r, R_{f} ; \Delta_{0}\right)+O(\log r)
$$

et

$$
(q-2) T(r, \Delta) \leqq 3 \sum_{i=1}^{q} N\left(2 r, a_{i} ; \Delta_{0}\right)+3 N\left(2 r, R_{f} ; \Delta_{0}\right)+O\left((\log r)^{2}\right) .
$$

Démonstration. Soient $h=\sqrt{2}, r_{\nu}=h^{\nu}(\nu=0,1,2,3, \cdots)$, pour $\nu \geqq 2$

$$
\begin{aligned}
& \Delta_{\nu}=\left\{z ;|\arg z| \leqq \alpha \text { et } r_{\nu-1} \leqq|z| \leqq r_{\nu}\right\} \\
& \Delta_{\nu}^{0}=\left\{z ;|\arg z| \leqq \alpha_{0} \text { et } r_{\nu-2} \leqq|z| \leqq r_{\nu+1}\right\},
\end{aligned}
$$

$R_{f}^{0}(\nu)$ (ou $\left.R_{f}(\nu)\right)$ la partie de $R_{f}$ sur $\Delta_{\nu}^{0}$ (ou $\Delta_{\nu}$ ),

$$
S_{\nu}=\frac{1}{\pi} \iint_{R_{f}(\nu)}\left(\frac{\left|f^{\prime}\left(r e^{i \varphi}\right)\right|}{1+\left|f\left(r e^{i \varphi}\right)\right|^{2}}\right)^{2} r d r d \varphi
$$

$n_{\nu}^{0}$ le nombre des points de $R_{f}^{0}(\nu)$ tels que ses images par $f(z)$ sur $w$-sphère sont $a_{1}, a_{2}, \cdots, a_{q}$ et $n_{\nu}^{1}$ le nombre des points ramifiés sur $R_{f}^{0}(\nu)$.

Représentons $\Delta_{\nu}^{0}$ conformément sur $|\zeta|<1$ comme le centre de $\Delta_{\nu}^{0}$ correspond à 0 , alors il existe un constant $\kappa$ tel que l'image de $\Delta_{\nu}$ est contenu dans $|\zeta|<\kappa$ pour tout $\nu$ : Puisque $S_{\nu}$ n'est pas changé par une transformation conforme, en appliquant le lemme 2 , on a

$$
(q-2) S_{\nu} \leqq n_{\nu}^{0}+n_{\nu}^{1}+\frac{A}{1-\kappa}
$$

et

$$
\begin{aligned}
(q-2) \sum_{\nu=2}^{n} S_{\nu} & \leqq \sum_{\nu=2}^{n} n_{\nu}^{0}+\sum_{\nu=2}^{n} n_{\nu}^{1}+O(n) \\
& =\sum_{\nu=2}^{n} n_{\nu}^{0}+\sum_{\nu=2}^{n} n_{\nu}^{1}+O\left(\log r_{n}\right) .
\end{aligned}
$$

En utilisant

$$
\sum_{\nu=2}^{n} S_{\nu}=S\left(r_{n}, \Delta\right)-S\left(r_{1}, \Delta\right), \quad \sum_{\nu=2}^{n} n_{\nu}^{0} \leqq 3 \sum_{i=1}^{q} n\left(r_{n+1}, a_{i} ; \Delta_{0}\right)
$$


et

$$
\sum_{\nu=2}^{n} n_{\nu}^{1} \leqq 3 n\left(r_{n+1}, R_{f} ; \Delta_{0}\right)
$$

on a

$$
(q-2) S\left(r_{n}, \Delta\right) \leqq 3 \sum_{i=1}^{q} n\left(r_{n+1}, a_{i} ; \Delta_{0}\right)+3 n\left(r_{n+1}, R_{f} ; \Delta_{0}\right)+O\left(\log r_{n}\right)
$$

Pour tout $r$, il y a un $n$ tel que $r_{n-1} \leqq r<r_{n}$. Pour ce $n$,

$$
S(r, \Delta) \leqq S\left(r_{n}, \Delta\right)
$$

et

$$
r_{n+1}=h^{2} r_{n-1}=2 r_{n-1}<2 r
$$

par conséquent

$$
(q-2) S(r, \Delta) \leqq 3 \sum_{i=1}^{q} n\left(2 r, a_{i} ; \Delta_{0}\right)+3 n\left(2 r, R_{f} ; \Delta_{0}\right)+O(\log r)
$$

et

$$
(q-2) T(r, \Delta) \leqq 3 \sum_{i=1}^{q} N\left(2 r, a_{i} ; \Delta_{0}\right)+3 N\left(2 r, R_{f} ; \Delta_{0}\right)+O\left((\log r)^{2}\right)
$$

Comme une conséquence de ce lemme, on a

THÉorème 3. Soient $f(z)$ une fonction algébroìde d'ordre $\rho>0$ définie par (1), $R_{f}$ la surface de recouvrement de $|z|<+\infty$ définie par $f(z)$. Si l'ordre des points ramifiés de $R_{f}$ est moins de celui de $f(z)$, alors il existe une direction $J$ : $\arg z=\theta_{0}$ telle que, pour $\varepsilon$ positif quelque soit petit, il existe au plus deux valeurs exceptionnelles au sens de Borel dans $\Delta_{\varepsilon}\left(\theta_{0}\right)=\left\{z ;\left|\arg z-\theta_{0}\right|<\varepsilon\right\}$ pour $f(z)$.

Démonstration. Il existe une direction $J: \arg z=\theta_{0}$ telle que l'ordre de $T\left(r, \Delta_{\varepsilon}\left(\theta_{0}\right)\right)$ est $\rho$ pour $\varepsilon$ positif quelconque. Soit $\varepsilon_{1}$ un nombre quelconque plus grand que $\varepsilon$. En appliquant le lemme 3 pour $\Delta_{\varepsilon}\left(\theta_{0}\right)$ et $\Delta_{\varepsilon_{1}}\left(\theta_{0}\right)$, s'il y a au moins trois valeurs exceptionnelles au sens de Borel dans $\Delta_{\varepsilon_{1}}\left(\theta_{0}\right)$, par l'hypothèse, l'ordre de $T\left(r, \Delta_{\varepsilon}\left(\theta_{0}\right)\right)$ devienne plus petit que $\rho$. C'est une contradiction. On a le résultat.

\section{Amélioration du théorème de Rauch.}

Dans ce paragraphe, on améliore et généralise le théorème de Rauch qui est cité dans l'introduction. 
On utilise les mêmes notations dans le paragraphe précédent et on met $\Delta_{\varepsilon}(\theta)=\{z ;|\arg z-\theta|<\varepsilon\}$ et $E=\left\{\theta ; 0 \leqq \theta \leqq 2 \pi\right.$ et $T\left(r, \Delta_{\varepsilon}(\theta), f\right)$ est d'ordre $\rho$ pour tout $\varepsilon>0\}$, où $f(z)$ est une fonction algébroïde dans $|z|<+\infty$ à $n$ branches d'ordre $\rho$ définie par (1).

Lemme 4. L'ensemble $E$ est fermé, par conséquent, il est compact.

Démonstration. Soit $\left\{\theta_{n}\right\}_{n=1}^{\infty}$ une suite quelconque qui converge vers $\theta_{0}$ et qui est contenue dans $E$. On démontre que $\theta_{0}$ appartient à $E$. Pour un $\varepsilon>0$ quelconque, il existe un $n_{0}$ tel que pour tout $n$ plus grand que $n_{0}$, on a

$$
T\left(r, \Delta_{\varepsilon}\left(\theta_{0}\right)\right) \geqq T\left(r, \Delta_{\varepsilon^{\prime}}\left(\theta_{n}\right)\right),
$$

si $\varepsilon^{\prime}$ est suffisamment petit. Cette inégalité signifie que $\theta_{0}$ appartient à $E$. Cela veut dire que $E$ est fermé.

THÉORÈme 4. Soit $f(z)$ une fonction algébroïde dans $|z|<+\infty$ d'ordre $0<\rho<+\infty$ définie par (1). Alors, on a le résultat suivant:

1) Le cas où $E$ se compose d'un nombre fini d'éléments.

Il existe une direction $J: \arg z=\theta_{0}$ telle que, pour $\varepsilon$ positif quelconque $f(z)$ admet au plus un nombre fini des valeurs exceptionnelles au sens de Borel dans $\Delta_{\varepsilon}\left(\theta_{0}\right)$.

2) Les autres cas.

Il existe une direction $J: \arg z=\theta_{0}^{\prime}$ telle que, pour $\varepsilon$ positif quelconque, $f(z)$ admet au plus dénombrablement infini des valeurs exceptionnelles au sens de Borel dans $\Delta_{\varepsilon}\left(\theta_{0}^{\prime}\right)$.

Démonstration. 1) Soit $E=\left\{\theta_{1}, \theta_{2}, \cdots, \theta_{\alpha}\right\}$ et $V(r)=r^{\rho(r)}$ où $\rho(r)$ est l'ordre précisé de $T(r, f)$. Il admet les propriétés suivantes: i) $\lim _{r \rightarrow \infty} \rho(r)=\rho$, ii) $\lim _{r \rightarrow \infty} \frac{V(k r)}{V(r)}=k \quad$ où $\quad k>1, \quad$ iii) $\quad V(r) \geqq T(r, f)$ pour tout $r$, iv) $\lim _{r \rightarrow \infty} \sup T(r, f) / V(r)=1$, v) $\rho(r)$ est continue, dérivable à droite et à gauche de quelque point et $\lim _{r \rightarrow \infty} \rho^{\prime}(r) r \log r=0$ (Voir Valiron [19]).

Pour $\varepsilon$ un nombre positif quelconque, on a

$$
T(r, f) \leqq \sum_{i=1}^{\alpha} T\left(r, \Delta_{\varepsilon}\left(\theta_{i}\right)\right)+T\left(r, \Delta^{\prime}\right)
$$

où $\Delta^{\prime}=\left\{\bigcup_{i=1}^{\alpha} \Delta_{\varepsilon}\left(\theta_{i}\right)\right\}^{C}$. Alors $T\left(r, \Delta^{\prime}\right)$ est d'ordre inférieur à $\rho . \quad$ En divisant les deux cotés de (6), on a 


$$
\frac{T(r, f)}{V(r)} \leqq \sum_{i=1}^{\alpha} \frac{T\left(r, \Delta_{\varepsilon}\left(\theta_{i}\right)\right)}{V(r)}+\frac{T\left(r, \Delta^{\prime}\right)}{V(r)}
$$

et puis, en considérant les limites supérieures des deux cotés, on a

$$
1=\lim _{r \rightarrow \infty} \sup \frac{T(r, f)}{V(r)} \leqq \sum_{i=1}^{\alpha} \lim \sup _{r \rightarrow \infty} \frac{T\left(r, \Delta_{\varepsilon}\left(\theta_{i}\right)\right)}{V(r)}
$$

parce que $T(r, \Delta)$ est d'ordre inférieur à $\rho$. Par conséquent, il existe un $i_{0}$ tel que

$$
\limsup _{r \rightarrow \infty} \frac{T\left(r, \Delta_{\varepsilon}\left(\theta_{i_{0}}\right)\right)}{V(r)} \geqq \frac{1}{\alpha}
$$

En appliquant le lemme 3 dans le paragraphe 3 pour $\Delta_{\varepsilon}\left(\theta_{i_{0}}\right)$, on a

$$
(q-2) T\left(r, \Delta_{\varepsilon}\left(\theta_{i_{0}}\right)\right) \leqq 3 \sum_{i=1}^{q} N\left(2 r, a_{i} ; \Delta_{\varepsilon}^{0}\right)+3 N\left(2 r, R_{f} ; \Delta_{\varepsilon}^{0}\right)+O\left((\log r)^{2}\right)
$$

où $\Delta_{\varepsilon}^{0}$ est au lieu de $\Delta_{0}$ dans le lemme 3 .

En divisant les deux cotés par $V(r)$ et prenant les limites supérieures, on a

$$
\begin{gathered}
(q-2) \varlimsup_{r \rightarrow \infty} \frac{T\left(r, \Delta_{\varepsilon}\left(\theta_{i_{0}}\right)\right)}{V(r)} \leqq 3 \sum_{i=1}^{q} \varlimsup_{r \rightarrow \infty} \frac{N\left(2 r, a_{i} ; \Delta_{\varepsilon}^{0}\right)}{V(r)}+3 \varlimsup_{r \rightarrow \infty} \frac{N\left(2 r, R_{f} ; \Delta_{\varepsilon}^{0}\right)}{V(r)} \\
\leqq 3 \sum_{i=1}^{q} \varlimsup_{r \rightarrow \infty} \frac{N\left(2 r, a_{i} ; \Delta_{\varepsilon}^{0}\right)}{V(r)}+3 \varlimsup_{r \rightarrow \infty} \frac{N\left(2 r, R_{f} ; \Delta_{\varepsilon}^{0}\right)}{V(2 r)} \varlimsup_{r \rightarrow \infty} \frac{V(2 r)}{V(r)},
\end{gathered}
$$

grâce au théorème du point ramifié,

$$
\begin{aligned}
& \leqq 3 \sum_{i=1}^{q} \varlimsup_{r \rightarrow \infty} \frac{N\left(2 r, a_{i} ; \Delta_{\varepsilon}^{0}\right)}{V(r)}+3(2 n-2) \varlimsup_{r \rightarrow \infty} \frac{T(2 r, f)}{V(2 r)} \varlimsup_{r \rightarrow \infty} \frac{V(2 r)}{V(r)} \\
& =3 \sum_{i=1}^{q} \varlimsup_{r \rightarrow \infty} \frac{N\left(2 r, a_{i} ; \Delta_{\varepsilon}^{0}\right)}{V(r)}+3(2 n-2) 2^{\rho} .
\end{aligned}
$$

Si $a_{1}, a_{2}, \cdots, a_{q}$ sont les valeurs exceptionnelles au sens de Borel dans $\Delta_{\varepsilon}^{0}$, on a

$$
(q-2) \frac{1}{\alpha} \leqq 3(2 n-2) 2^{\rho},
$$

c'est-à-dire

$$
q \leqq 2+3 \alpha 2^{\rho}(2 n-2),
$$

de sorte que le nombre des valeurs exceptionnelles au sens de Borel dans $\Delta_{\varepsilon}^{0}$ est au plus $2+\left[3 \alpha 2^{\rho}(2 n-2)\right]$. 
L'indice $i_{0}$ est dépendent de $\varepsilon$, mais $i_{0}$ est au plus $\alpha$, par conséquent, pour une suite $\varepsilon_{1}>\varepsilon_{2}>\varepsilon_{3}>\cdots>\varepsilon_{m}>\cdots \rightarrow 0$, il existe un $i_{0}=i_{0}\left(\varepsilon_{m}\right)$ $m=1,2,3, \cdots$ Dans les discussions précédentes, si on prend

$$
\Delta_{\varepsilon_{m}}^{0}=\Delta_{2 \varepsilon_{m}}\left(\theta_{i_{0}}\right) \quad m=1,2,3, \cdots,
$$

on a le résultat.

2) On démotre ici le cas où $E$ n'est pas fini. Soient $\varepsilon>0$ quelconque fixe et $\theta$ un élément de $E$. Soit $E^{\prime}$ un ensemble sur $|z|=1$ tel que $e^{i \theta}$ appartient a $E^{\prime}$ quand $\theta$ appartient a $E$, alors $E^{\prime}$ est compact et $E^{\prime} \subset \subset_{\theta \in E} \Delta_{\varepsilon}(\theta)$, par conséquent, il existe un nombre fini de $\theta$, soient $\theta_{1}, \theta_{2}, \cdots, \theta_{\beta(\varepsilon)}$ tels que

$$
E^{\prime} \subset \bigcup_{i=1}^{\beta} \Delta_{\varepsilon}\left(\theta_{i}\right)
$$

où $\beta=\beta(\varepsilon)$.

D'aprés 1) dans cette démonstration, il existe un $i_{0}$ tel que le nombre des valeurs exceptionnelles au sens de Borel dans $\Delta_{2 \varepsilon}\left(\theta_{i_{0}}\right)$ est au plus $2+\left[3 \beta 2^{\rho}(2 n-2)\right]$. Pour une suite $\varepsilon_{1}>\varepsilon_{2}>\cdots>\varepsilon_{m}>\cdots \rightarrow 0$, on a une suite de $\theta:\left\{\theta_{m}\right\}_{m=1}^{\infty}$ qui admet la propriété précédente. Puisque $E$ est fermé, il existe au moins un point d'accumulation de $\left\{\theta_{m}\right\}_{m=1}^{\infty}$ dans $E$. Soit $\theta_{0}^{\prime}$ un tel point. On peut supposer que $\theta_{m}$ converge vers $\theta_{0}^{\prime}$. Il existe un $m_{0}$ tel que pour tout $m$ plus grand que $m_{0}$,

$$
\Delta_{\varepsilon}\left(\theta_{0}^{\prime}\right) \supset \Delta_{\varepsilon_{m}}\left(\theta_{m}\right) .
$$

Cela veut dire que le nombre des valeurs exceptionnelles au sens de Borel dans $\Delta_{\varepsilon}\left(\theta_{0}^{\prime}\right)$ est au plus $2+\left[3 \beta_{m} 2^{\rho}(2 n-2)\right]$ où $\beta_{m}=\beta\left(\varepsilon_{m}\right)$. Soit $\beta^{\prime}=\lim _{m \rightarrow \infty} \beta_{m}$, alors, le nombre des valeurs exceptionnelles au sens de Borel dans $\Delta_{\varepsilon}\left(\theta_{0}^{\prime}\right)$ est au plus $2+\left[3 \beta^{\prime} 2^{\rho}(2 n-2)\right]$, qui est fini ou dénombrablement infini.

\section{Relations entre l'ensemble d'adhérence fine et la distribu-} tion des valeurs d'une fonction algébroïde.

Dans ce paragraphe, on donne quelques résultats un peu différents des théorèmes précédents.

Soit $\tilde{C}_{f}(\infty)$ l'ensemble d'adhérence fine de $f(z)$ en $\infty$ qui est défini comme suivant: Soit $V=\{v$; voisinage fin de $\infty$ dans le plan $\}$, alors

$$
\widetilde{C}_{f}(\infty)=\bigcap_{v \in V} \overline{f\left(v^{\prime}\right)}
$$


où $v^{\prime}$ est l'ensemble le plus grand sur $R_{f}$ dont la projection sur le plan est $v$. L'ensemble $\tilde{C}_{f}(\infty)$ est non-vide, compact dans la sphère de Riemann ([11]). Alors, on sait que 1) $\tilde{C}_{f}(\infty)$ est total ou se compose d'au plus $n$ points et dans ce cas $f(z)$ n'a pas de valeurs exceptionnelles au sens de Picard; 2) si $\widetilde{C}_{f}(\infty)$ ne contient pas $\infty$, chaque coefficient de (1) admet une limite fine finie ([11]). En utilisant ce fait, on a le

ThÉORÈme 5. Soit $f(z)$ une fonction algébroïde dans $|z|<+\infty$ à $n$ branches définie par (1). Si $\tilde{C}_{f}(\infty)$ n'est pas total, il existe $m(\leqq n)$ directions $J_{1}$ : $\arg z=\theta_{1}$, $\cdots, J_{m}$ : arg $z=\theta_{m}$ telles que $f(z)$ n'a pas de valeurs exceptionnelles au sens de Picard dans

$$
\Delta_{\varepsilon}=\bigcup_{i=1}^{m} \Delta_{\varepsilon}\left(\theta_{i}\right) \quad \text { ò̀ } \quad \Delta_{\varepsilon}\left(\theta_{i}\right)=\left\{z ;\left|\arg z-\theta_{i}\right|<\varepsilon\right\}
$$

pour $\varepsilon$ positif quelconque et $m$ est le nombre des éléments de $\widetilde{C}_{f}(\infty)$ moins le nombre d'éléments de $\widetilde{C}_{f}(\infty)$ tels que

$$
A_{\alpha_{0}}(z)=\alpha_{0}^{n}+a_{1}(z) \alpha_{0}^{n-1}+\cdots+a_{n}(z)
$$

est rationnelle où $\alpha_{0}$ appartient à $\tilde{C}_{f}(\infty)$.

Démonstration. On peut supposer que $\widetilde{C}_{f}(\infty)$ ne contient pas l'infini en considérant quelque transformation linéaire. Soient $\alpha_{1}, \alpha_{2}, \cdots, \alpha_{m}$ les éléments de $\tilde{C}_{f}(\infty)$ tels que

$$
A_{i}(z)=\alpha_{i}^{n}+a_{1}(z) \alpha_{i}^{n-1}+\cdots+a_{n}(z)
$$

n'est pas rationnelle $(i=1,2, \cdots, m)$. Soient $b_{n} e^{i x_{n}}$ et $d_{n} e^{i y_{n}}(n=1,2, \cdots)$ les zéros et les pôles de $A_{i}(z)$ respectivement. Alors il existe une valeurs $\theta_{i}$ qui est un point d'accumulation de $\left\{x_{n}\right\}$ et $\left\{y_{n}\right\}$ à la fois ([11]). $A_{i}(z)$ admet la limite fine 0 en $\infty$. Soient $J_{i}: \arg z=\theta_{i}, i=1,2, \cdots, m$, alors ces $J_{i}$ sont les directions que l'on cherche. En effet, soit $\alpha \notin \widetilde{C}_{f}(\infty)$, alors

$$
A_{\alpha}(z)=\alpha^{n}+a_{1}(z) \alpha^{n-1}+\cdots+a_{n}(z)
$$

admet la limite fine finie $\beta_{\alpha} \neq 0 . \quad$ Par conséquent

$$
A_{\alpha}^{-1}\left(\left|w-\beta_{\alpha}\right|>r\right) \text { où } 0<r<\left|\beta_{\alpha}\right|
$$

est effilé en $\infty$, contient toutes les pôles de $A_{\alpha}(z)$ sauf un nombre fini et la somme des angles vu de l'origine de $A_{\alpha}^{-1}\left(\left|w-\beta_{\alpha}\right|>r\right) \cap(|z|>l)$ tend vers 0 pour $l \rightarrow+\infty$ quand $A_{\alpha}(z)$ n'est pas rationnelle ([10]). Si $A_{\alpha}(z)$ est rationnelle, $\alpha$ est pris par $f(z)$ où $f(z)$ est $\infty$ grâce à l'égalité 


$$
A_{\alpha}(z)=\prod_{i=1}^{n}\left(\alpha-f_{i}(z)\right)
$$

où $f_{i}(z)(i=1,2, \cdots, n)$ sont les branches de $f(z)$, de sorte que $\alpha \neq \alpha_{1}$, $\alpha_{2}, \cdots, \alpha_{m}, \infty$ est pris par $f(z)$ l'infini fois dans $\Delta_{\varepsilon}$ exprimé dans le théorème. $\alpha_{1}, \alpha_{2}, \cdots, \alpha_{m}$ et $\infty$ sont pris par $f(z)$ dans $\Delta_{\varepsilon}$ l'infini fois grâce à sa définition. On a le résultat.

Le " $m$ " dans ce théorème ne peut pas être repetissé en général. En effet, on donne un exemple.

Exemple. Soit $f_{i}(z)$ une fonction méromorphe non rationnelle dans $|z|<+\infty$ admettant la limite fine 0 en $\infty$ telle que sa toute pôle est sur $\arg z=\theta_{i}(i=1,2, \cdots, n)$ où $\theta_{i} \neq \theta_{j}$ si $i \neq j$. Pour $n$ valeurs $u_{1}, u_{2}, \cdots$, $u_{n}$ différentes les unes les autres, on résolve les équations

$$
u_{i}^{n}+a_{1}(z) u_{i}^{n-1}+\cdots+a_{n}(z)=f_{i}(z) ; i=1,2, \cdots, n,
$$

par rapport aux $a_{1}(z), a_{2}(z), \cdots, a_{n}(z)$. On définie une fonction algébroïde $f(z)$ par

$$
f^{n}+a_{1}(z) f^{n-1}+\cdots+a_{n}(z)=0 \text {. }
$$

Alors, $\tilde{C}_{f}(\infty)=\left\{u_{1}, u_{2}, \cdots, u_{n}\right\} ; J_{1}, J_{2}, \cdots, J_{n}$ sont différentes les unes les autres et on ne peut pas repetisser " $n$ ", puisque si on le repetisse, au moins une valeur exceptionnelle apparaît dans $\Delta_{\varepsilon}$.

Corollaire 2. Soit $f(z)$ une fonction algébroide dans $|z|<+\infty$ à $n$ branches definie par (1). Si $\tilde{C}_{f}(\infty)$ n'est pas total, $f(z)$ admet une direction de Julia.

\section{Théorème de type wimanien.}

Pour une fonction algébroïde dans $|z|<+\infty$, le théorème de type wimanien n'existe pas généralement. On donne, ici, une condition suffisante pour qu'un théorème de type wimanien soit vrai.

Lemme 5. Soit $\varphi(z)$ une fonction entière d'ordre $0<\rho<1 / 2$ et $\varepsilon$ un nombre positif quelconque plus petit que $\rho$. Alors il existe une suite $\left\{r_{k}\right\}_{k=1}^{\infty}$ telle que $r_{k} \uparrow+\infty$ et $\log m\left(r_{k}\right) \geqq r_{k}^{\rho-\varepsilon}$ où $m(r)=\min _{|\boldsymbol{z}|=r}|\varphi(z)|$. (Voir [8]).

\section{LEMME 6. Soit}




$$
z^{n}+a_{1} z^{n-1}+\cdots+a_{n}=0
$$

une équation algébrique telle que $a_{n} \neq 0$. Alors les racines $z_{1}, z_{2}, \cdots, z_{n}$ de cette équation satisfont à

$$
\min \left(\sqrt[n]{\frac{\left|a_{n}\right|}{n}}, n-1 \sqrt{\frac{\left|a_{n}\right|}{n\left|a_{1}\right|}}, \cdots, \frac{\left|a_{n}\right|}{n\left|a_{n-1}\right|}\right) \leqq \min _{1 \leqq i \leqq n}\left|z_{i}\right|
$$

Démonstration. On sait que

$$
\max _{1 \leqq i \leqq n}\left|z_{i}\right| \leqq \max \left(n\left|a_{1}\right|, \sqrt{n\left|a_{2}\right|}, \cdots, \sqrt[n]{n\left|a_{n}\right|}\right) .
$$

Or, $1 / z_{i}(i=1,2, \cdots, n)$ sont les racines de l'équation

$$
z^{n}+\frac{a_{n-1}}{a_{n}} z^{n-1}+\cdots+\frac{a_{1}}{a_{n}} z+\frac{1}{a_{n}}=0 .
$$

En appliquant (8) pour cette équation, on a

$$
\max _{1 \leqq i \leqq n}\left|1 / z_{i}\right| \leqq \max \left(\frac{n\left|a_{n-1}\right|}{\left|a_{n}\right|}, \sqrt{\frac{n\left|a_{n-2}\right|}{\left|a_{n}\right|}}, \cdots, n \sqrt{\frac{n}{\left|a_{n}\right|}}\right),
$$

par conséquent,

$$
\min \left(\sqrt[n]{\frac{\left|a_{n}\right|}{n}}, \quad n-1 \sqrt{\frac{\left|a_{n}\right|}{n\left|a_{1}\right|}}, \cdots, \frac{\left|a_{n}\right|}{n\left|a_{n-1}\right|}\right) \leqq \min _{1 \leqq i \leqq n}\left|z_{i}\right| .
$$

THÉORÈme 6. Soit $f(z)$ une fonction algébroïde entière, c'est-à-dire, tous coefficients de (1) sont entiers, d'ordre $0<\rho<1 / 2$. Si $a_{n}(z)$ est d'ordre $\rho$ et si d'autres coefficients sont d'ordre moins de $\rho$, alors, il existe une suite $\left\{r_{k}\right\}_{k=1}^{\infty}$ telle que $r_{k} \uparrow+\infty$ et $|f(z)| \rightarrow+\infty$ sur $|z|=r_{k}$ quand $k$ tend vers $+\infty$.

Démonstration. Soient $\rho_{1}, \rho_{2}, \cdots, \rho_{n-1}$ les ordres de $a_{1}(z), a_{2}(z), \cdots, a_{n-1}(z)$ respectivement, alors, par l'hypothèse, $\rho_{i}<\rho(i=1,2, \cdots, n-1)$. Soit $\varepsilon>0$ tel que $\rho_{i}+\varepsilon<\rho-\varepsilon$ pour tout $i$. D'après la définition d'ordre d'une fonction entière, il existe un $r_{0}$ tel que pour tout $r$ plus grand que $r_{0}$,

$$
\log M_{i}(r) \leqq r^{\rho_{i}+\varepsilon}
$$

où $M_{i}(r)=\max _{|z|=r}\left|a_{i}(z)\right|(i=1,2, \cdots, n-1)$.

Grâce au lemme 5 , il existe une suite $\left\{r_{k}\right\}_{k=1}^{\infty}$ telle que $r_{k} \uparrow \infty$ et $\log m_{n}\left(r_{k}\right) \geqq r_{k}^{\rho-\varepsilon}$ pour tout $k$ où $m_{n}(r)=\min _{|z|=r}\left|a_{n}(z)\right|$. 
Il y a un $k_{0}$ tel que pour tout $k$ plus grand que $k_{0}$, on a

$$
\log M_{i}\left(r_{k}\right) \leqq r_{k}^{\rho_{i}+\varepsilon}<r_{k}^{\rho-\varepsilon} \leqq \log m_{n}\left(r_{k}\right),
$$

de sorte que, sur $|z|=r_{k}$

$$
\frac{M_{i}\left(r_{k}\right)}{m_{n}\left(r_{k}\right)} \leqq \exp \left(-r_{k}^{\rho-\varepsilon}+r_{k}^{\rho_{i}+\varepsilon}\right)=\exp \left(-r_{k}^{\rho-\varepsilon}\left(1-r_{k}^{\rho_{i}-\rho+2 \varepsilon}\right)\right) .
$$

Puisque $\rho_{i}+2 \varepsilon-\rho<0$, le terme dernier tend vers 0 quand $k$ tend vers $\infty$ pour tout $i$. En conséquence, sur $|z|=r_{k}$,

$$
\sqrt[i]{\frac{\left|a_{n}(z)\right|}{n\left|a_{n-i}(z)\right|}} \rightarrow+\infty
$$

quand $k$ tend vers $\infty$ pour tout $i$. En vertu du lemme 6,

$$
|f(z)| \rightarrow+\infty \text { sur }|z|=r_{k}
$$

quand $k$ tend vers $\infty$.

N.B. Ce théorème n'est pas vrai toujours même si l'ordre de $f(z)$ est moins de $1 / 2$.

\section{Algébroïdes dans le cercle unité.}

Soit $f(z)$ une fonction algébroïde d'ordre $\rho$ dans $|z|<1$ définie par

$$
f^{n}+a_{1}(z) f^{n-1}+\cdots+a_{n}(z)=0
$$

où $a_{1}(z), \cdots, a_{n}(z)$ sont méromorphes dans $|z|<1$.

On sait que, en utilisant les mêmes symboles dans le paragraphe 1,

$$
T(r, f)=T(r, w)+O(1)
$$

pour tout $w$ et

$$
(q-2) T(r, f) \leqq \sum_{i=1}^{q} N\left(r, w_{i}\right)+N\left(r, R_{f}\right)+S(r)
$$

où $w_{1}, w_{2}, \cdots, w_{q}$ sont $q(\geqq 3)$ points de la sphère de Riemann et $S(r)=$ $O(\log T(r, f))+O(\log 1 /(1-r))$ sauf un ensemble $\Delta_{r} \operatorname{de} r$ tel que $\int_{\Delta_{r}} d(1 /(1-r))<+\infty$; en conséquence, $f(z)$ admet au plus $2 n$ valeurs exceptionnelles au sens de Borel quand $\rho$ est positif.

On considère dans ce paragraphe une précision du dernier résultat précédent. Pour cet effet, on donne une définition: 
Le point $z_{0}=e^{i \theta_{0}}$ est un point de Borel pour $f(z)$ si et seulement si, pour tout $\varepsilon>0, N\left(r, w ; \Delta_{\varepsilon}\left(\theta_{0}\right)\right)$ est d'ordre $\rho$ sauf au plus $2 n$ valeurs de $w$, quand l'ordre de $f(z)$ est $\rho$ positif où $\Delta_{\varepsilon}\left(\theta_{0}\right)=\left\{z ;|z|<1\right.$ et $\left.\left|\arg z-\theta_{0}\right|<\varepsilon\right\}$.

Pour les fonctions méromorphes dans $|z|<1$ d'ordre positif, Valiron [17] a donné la définition du point de Borel et obtenu beaucoup de résultats; surtout, il a trouvé qu'une fonction méromorphe d'ordre fini $\rho>0$ dans $|z|<1$ admet au moins un point de Borel sur la circonférence de ce cercle.

Ici, on donne quelques conditions suffisantes pour qu'une fonction algébroïde admette au moins un point de Borel et un autre résultats analogue au théorème 4 dans le paragraphe 4 sans démonstrations parce que l'on peut démontrer de la même manière.

LEMME 7. Soit $h(z)$ une fonction méromorphe d'ordre fini $\rho>0$ dans $|z|<1$. Alors, il $y$ a un point $z_{0}$ sur $|z|=1$ et une droite $J$ passant par $z_{0}$, qui peut être confondue avec la tangente au cercle, tels que, soit $\Delta$ un domaine angulaire arbitraire qui contient $J$ et qui est borné par deux droites passant par $z_{0}, g(z)$ une fonction méromorphe dans $|z|<1$ et $\left\{z_{\nu}(h=g ; \Delta)\right\}$ les zéros de $h(z)-g(z)$ dans $\Delta$ sans considération de multiplicité, si $g(z)$ est d'ordre inférieur à $\rho$, alors

$$
\sum_{\nu=1}^{\infty}\left(1-\left|z_{\nu}(h=g ; \Delta)\right|\right)^{\rho+1-\varepsilon}=\infty, \quad \varepsilon>0
$$

avec deux exceptions possibles pour $g(z)$; et si $h(z)$ est de type divergent et $\int_{0}^{1} T(r, g)(1-r)^{\rho-1} d r<+\infty$, alors

$$
\sum_{\nu=1}^{\infty}\left(1-\left|z_{\nu}(h=g ; \Delta)\right|\right)^{\rho+1}=\infty
$$

avec deux exceptions possibles pour $g(z)$. (Voir Tsuji [12].)

En appliquant ce lemme comme dans la démonstration du théorème 1 du paragraphe 3 , on trouve

ThÉonème 7. Soit $f(z)$ une fonction algébrö̈de d'ordre fini $\rho>0$ définie par (9). Si un seul des $a_{1}(z), \cdots, a_{n}(z)$ est d'ordre $\rho$ et d'autres sont d'ordre inférieur $\grave{a} \rho$, alors $i l$ existe un point $z_{0}$ sur $|z|=1$ et une droite $J$ passant par $z_{0}$, et qui peut être confondue avec la tangente au cercle, tels que, soient $\Delta$ un domaine angulaire arbitraire qui contient $J$ et qui est borné par deux droites passant par $z_{0}$ et $\left\{z_{\nu}(a)\right\}$ (ou $\left.\left\{z_{\nu}(\infty)\right\}\right)$ les zéros de $f(z)-a$ (ou les pôles de $f(z)$ ) dans 4 , alors

$$
\sum_{\nu=1}^{\infty}\left(1-\left|z_{\nu}(a)\right|\right)^{\rho+1-\varepsilon}=\infty, \quad \varepsilon>0
$$


sauf $2 n$ valeurs de a au plus; de plus si $f(z)$ est de type divergent et si un seul des coefficients est de type divergent d'ordre $\rho$ et d'autres sont de type convergent d'ordre $\rho$ au plus, on peut changer (13) comme

$$
\sum_{\nu=1}^{\infty}\left(1-\left|z_{\nu}(a)\right|\right)^{\rho+1}=\infty
$$

sauf $2 n$ valeurs de a au plus.

N.B. Le point dans ce théorème est un point de Borel pour $f(z)$.

On a aussi

ThÉORÈme 8. Soit $f(z)$ une fonction algébroïde d'ordre fini $\rho>0$ dans $|z|<1$ définie par (9). S'il existe $n-1$ valeurs exceptionnelles formelles finies, il y a un point de Borel pour $f(z)$ sur la circonférence du cercle unité. Ici, une valeur u exceptionnelle formelle finie veut dire que l'ordre de

$$
F(u, z)=u^{n}+a_{1}(z) u^{n-1}+\cdots+a_{n}(z)
$$

est inférieur à $\rho$.

Puis, en appliquant le lemme 2 dans le paragraphe 3 et la méthode dans [12], on a

Lemme 8. Soient $w_{1}, w_{2}, \cdots, w_{q} q(\geqq 3)$ valeurs distinctes dans la sphère de Riemann, $\Delta=\{z ;|z|<1$ et $|\arg z-\theta|<\varepsilon\}, \Delta_{0}=\left\{z ;|z|<1\right.$ et $\left.|\arg z-\theta|<\varepsilon_{1}\right\}$ où $\varepsilon_{1}>\varepsilon>0$ et $f(z)$ une fonction algébrö̈de dans $\Delta_{0} \grave{a} k$ branches. Alors,

$$
(q-2) S(r, \Delta) \leqq 9 \sum_{i=1}^{q} n\left(\frac{r+3}{4}, w_{i} ; \Delta_{0}\right)+9 n\left(\frac{r+3}{4}, R_{f} ; \Delta_{0}\right)+O\left(\frac{1}{1-r}\right)
$$

et

$$
(q-2) T(r, \Delta) \leqq 63 \sum_{i=1}^{q} N\left(\frac{r+3}{4}, w_{i} ; \Delta_{0}\right)+63 N\left(\frac{r+3}{4}, R_{f} ; \Delta_{0}\right)+O\left(\log \frac{1}{1-r}\right) .
$$

Comme dans la démonstration du théorème 3 dans le paragraphe 3 , on a

THÉORÈme 9. Soient $f(z)$ une fonction algébroide d'ordre fini $\rho>0$ dans $|z|<1$ définie par (9), $R_{f}$ la surface de recouvrement de $|z|<1$ définie par $f(z)$. Si l'ordre des points ramifiés de $R_{f}$ est inférieur à $\rho$, alors, il existe un point de Borel $z_{0}=e^{i \theta_{0}}$ sur $|z|=1$ pour $f(z)$.

N.B. Dans ce cas, il existe au plus deux valeurs exceptionnelles au sens de Borel dans $\Delta_{\varepsilon}\left(\theta_{0}\right)$ pour $\varepsilon>0$ quelconque. 
On ne peut pas, à mon regret, démontrer s'il y a toujours au moins un point de Borel sur la circonférence du cercle unité quand l'ordre de $f(z)$ est positif. Ici, on donne un théorème qui approche de cette question, qui est analogue au théorème 4 dans le paragraphe 4 .

ThÉORÈme 10. Soient $f(z)$ une fonction algébroïde d'ordre fini $\rho>0$ dans $|z|<1$ définie par (9), $E=\left\{\theta ; 0 \leqq \theta \leqq 2 \pi, T\left(r, \Delta_{\varepsilon}(\theta)\right)\right.$ est d'ordre $\rho$ pour $\varepsilon$ quelconque positif $\}$ où $\Delta_{\varepsilon}(\theta)=\{z ;|z|<1$ et $|\arg z-\theta|<\varepsilon\}$. Alors,

1) Le cas où $E$ se compose d'un nombre fini d'éléments.

Il existe un point $z_{0}=e^{i \theta_{0}}$ sur $|z|=1$ tel que, pour tout $\varepsilon>0, f(z)$ admet au plus un nombre fini des valeurs exceptionnelles au sens de Borel dans $\Delta_{\varepsilon}\left(\theta_{0}\right)$.

2) Les autres cas.

Il existe un point $z_{0}^{\prime}=e^{i \theta_{0}^{\prime}}$ sur $|z|=1$ tel que, pour $\varepsilon>0$ quelconque, $f(z)$ admet au plus dénombrablement infini des valeurs exceptionnelles au sens de Borel dans $\Delta_{\varepsilon}\left(\theta_{0}^{\prime}\right)$.

On démontre ce théorème en utilisant l'ordre précisé $\rho\left(\frac{1}{1-r}\right)$ au lieu de $\rho(r)$ dans le paragraphe 4 .

\section{BiBLIOGRAPHIE}

[ 1 ] M. Biernacki, Sur les directions de Borel des fonctions méromorphes. Acta Math. 56 (1930), 197-204.

[2] J. Dufresnoy, Sur les domaines couverts par les valeurs d'une fonction méromorphe ou algébroïde. Ann. École Norm. Sup. (3) 58 (1941), 179-259.

[ 3 ] G. Julia, Leçons sur les fonctions uniformes à points singulier essentiel isolé. Paris, 1924.

[4] A. Ostrowski, Über Folgen analytischer Funktionen und einige Verschärfungen des Picardschen Satzes. Math. Zeit. 24 (1925), 215-258.

[5] A. Rauch, Extensions de théorèmes relatifs aux directions de Borel des fonctions méromorphes. Journ. de Math. 12 (1933), 109-171.

[6] A. Rauch, Sur les algébroïdes entières. C.R. Acad. Sci. Paris, 202 (1936), 2041-2043.

[ 7 ] G. Rémoundos, Extensions aux fonctions algébroïdes multiformes du théorème de Picard et de ses généralisations. Mém. Sci. Math. Paris, 1927.

[8] L. Sario-K. Noshiro, Value distribution theory. Van Nostrand, Princeton 1966.

[ 9 ] H.L. Selberg, Algebroide Funktionen und Umkehrfunktionen Abelscher Integrale. Avh. Norske Vid. Akad. Oslo 8 (1934), 1-72.

[10] N. Toda, Sur les fonctions méromorphes aux limites fines. Nagoya Math. Journ. 29 (1967), 61-68.

[11] N. Toda, Sur l'ensemble d'adhérence fine des fonctions algébroïdes. Nagoya Math. Journ. 30 (1967), 295-302.

[12] M. Tsuji, Potential theory in modern function theory. Maruzen, Tokyo 1959.

[13] Y. Tumura, Quelques appliquations de la théorie de M. Ahlfors. Japanese Journ. Math. 18 (1942-1943), 303-322. 
[14] E. Ullrich, Über den Einfluss der Verzweigtheit einer Algebroide auf ihre Wertverteilung. Journ. rei. u. ang. Math. 167 (1931), 198-220.

[15] G. Valiron, Recherche sur le théorème de M. Borel dans la théorie des fonctions méromorphes. Acta Math. 52 (1928), 67-92.

[16] G. Valiron, Sur la derivée des fonctions algébroïdes. Bull. Sci. Math. 59 (1931), 17-39.

[17] G. Valiron, Points de Picard et points de Borel des fonctions méromorphes dans un cercle. Bull. Sci. Math. 67 (1932), 10-32.

[18] G. Valiron, Sur les directions de Borel des fonctions algébroïdes méromorphes d'ordre infini. C. R. Acad. Sci. Paris, 206 (1938), 735-737.

[19] G. Valiron, Fonctions entières d'ordre fini et fonctions méromorphes. Mono. L'Enseign. Math. Genève No. 8 (1960).

Institut de Mathématiques,

Université de Tôhoku,

Sendai, Japon. 